\title{
Relação entre analgesia do parto e seus desfechos obstétricos
}

\author{
Labor analgesia related to obstetric outcomes \\ Relación entre analgesia laboral y resultados obstétricos
}

Ana Vitória Meireles Veiga

ORCID: https://orcid.org/0000-0003-2247-4824

Universidade Federal do Delta do Parnaíba, Brasil

E-mail: vitmei@hotmail.com

Ariane Oliveira Dinato

ORCID: https://orcid.org/0000-0002-1319-5746

Universidade Federal do Delta do Parnaíba, Brasil

E-mail: aneedinato@hotmail.com

Marinice Saraiva Attem

ORCID: https://orcid.org/0000-0002-7460-5788 Instituto de Educação Superior do Vale do Parnaíba, Brasil

E-mail: mariniceattem @gmail.com

Raysa Maria Silva de Araujo

ORCID: https://orcid.org/0000-0003-2246-0983 Instituto de Educação Superior do Vale do Parnaíba, Brasil

E-mail: rayssaaraujo93@hotmail.com

Luma Nunes Pereira da Silva

ORCID: https://orcid.org/0000-0003-2386-5325

Universidade Federal do Delta do Parnaíba, Brasil

E-mail: lumanps0804@gmail.com

Lucas de Carvalho Techi

ORCID: https://orcid.org/0000-0002-8226-7774

Universidade Federal do Delta do Parnaíba, Brasil E-mail: lucascarvalhotechi@hotmail.com

Eduardo de Carvalho Carneiro

ORCID: https://orcid.org/0000-0001-7202-0930 Instituto de Educação Superior do Vale do Parnaíba, Brasil

E-mail: lucascarvalhotechi@hotmail.com

Igor dos Santos Cavalcante

ORCID: https://orcid.org/0000-0003-2907-9026

Universidade Federal do Delta do Parnaíba, Brasil E-mail: igorsc@live.com

Lúcia Maria de Sousa Aguiar dos Santos ORCID: https://orcid.org/0000-0002-9316-2214 Centro Universitário UniFacid, Brasil E-mail: 1msasantos@gmail.com

\section{Resumo}

A questão do uso de analgesia no parto, mesmo não sendo adotada comumente no Brasil, tem ocupado grande relevância nas discussões a respeito de como tornar esse momento menos doloroso e com menos desfechos desfavoráveis, tanto pro recém-nascido quanto pra mãe. Objetivo: O presente artigo tem por objetivo avaliar a relação entre o uso das técnicas anestésicas e o desfecho obstétrico das gestantes durante o trabalho de parto, tendo como base os artigos publicados em base de dados. Métodos: Trata-se de uma revisão integrativa que visa analisar e sintetizar o que a literatura traz sobre a analgesia do parto e seus possíveis desfechos obstétricos. A busca de artigos foi feita nas seguintes bases de dados: Scientific Eletronic Library Online (SciELO) e no Literatura Latino-Americana e do Caribe (LILACS); além do portal PUBMED. Resultados e Discussão: Apesar de se apresentar satisfatória para controlar a dor, a administração de anestésicos locais pode desencadear diversos efeitos desfavoráveis. Durante a pesquisa destacou-se entre os estudos o aumento da duração do período expulsivo do trabalho de parto, a bradicardia fetal importante, além da elevação do número de partos instrumentais. Considerações finais: Este artigo considerou que existe o benefício do uso de analgesia para alívio da dor e conforto durante o parto, mas observou-se que existe uma relação entre seu uso e: o parto vaginal assistido, o atraso no início da lactação, o aumento na segunda fase de parto e a bradicardia fetal.

Palavras-chave: Parto; Analgesia; Dor de parto. 


\begin{abstract}
The issue of the use of analgesia in childbirth, even though it is not commonly adopted in Brazil, has been largely replaced in respect of how to make this moment less painful and with less unfavorable outcomes, both for the newborn and for the mother. Objective: This article aims to assess the relationship between the use of anesthetic techniques and the obstetric outcome of pregnant women during labor, based on articles published in the database. Methods: This is an integrative review that aims to analyze and synthesize that the literature brings about labor analgesia and its possible obstetric outcomes. The search for articles was carried out in the following databases: Scientific Eletronic Library Online (SciELO) and in Latin American and Caribbean Literature (LILACS); in addition to the PUBMED portal. Results and Discussion: Despite being satisfactory to control pain, the administration of local anesthetics can trigger several unfavorable effects. During a study, the length of the expulsive period of labor, an important fetal bradycardia, in addition to the increase in the number of instrumental deliveries, stood out among the studies. Final considerations: This article considers that there is a benefit of using analgesia for pain relief and comfort during childbirth, but it was observed that there is a relationship between its use and: assisted vaginal delivery, delay in the beginning of lactation, increase in the second stage of delivery and fetal bradycardia.
\end{abstract}

Keywords: Children; Analgesia; Children's pain.

\title{
Resumen
}

El tema del uso de analgesia en el parto, aunque no es comúnmente adoptado en Brasil, ha ocupado gran relevancia en las discusiones sobre cómo hacer que este momento sea menos doloroso y con resultados menos desfavorables, tanto para el recién nacido como para la madre. Objetivo: Este artículo tiene como objetivo evaluar la relación entre el uso de técnicas anestésicas y el resultado obstétrico de la gestante durante el trabajo de parto, a partir de artículos publicados en la base de datos. Métodos: Se trata de una revisión integradora que tiene como objetivo analizar y sintetizar lo que aporta la literatura sobre la analgesia del trabajo de parto y sus posibles resultados obstétricos. La búsqueda de artículos se realizó en las siguientes bases de datos: Scientific Eletronic Library Online (SciELO) y en Literatura Latinoamericana y Caribeña (LILACS); además del portal PUBMED. Resultados y Discusión: A pesar de ser satisfactoria para controlar el dolor, la administración de anestésicos locales puede desencadenar varios efectos desfavorables. Durante la investigación, se destacó entre los estudios el aumento en la duración del período expulsivo del parto, la importante bradicardia fetal, además del aumento en el número de partos instrumentales. Consideraciones finales: Este artículo consideró que existe un beneficio de usar analgesia para el alivio del dolor y el confort durante el parto, pero se observó que existe una relación entre su uso y: parto vaginal asistido, retraso en el inicio de la lactancia, aumento de la segunda etapa del parto y bradicardia fetal.

Palabras clave: Niños; Analgesia; Dolor de niños.

\section{Introdução}

O trabalho de parto é sabidamente um processo doloroso, em especial pelas diversas mudanças fisiológicas para o nascimento do bebê, tais como a dilatação cervical, o processo de contração uterina por meio do aumento da tensão das fibras musculares do útero, compressão das estruturas pélvicas e raízes do plexo lombo-sacro e tração ovariana e peritoneal (Penuela et al, 2019).

O estímulo nociceptivo promovido pela dor do trabalho de parto pode ser um empecilho para o curso natural do mecanismo do parto, bem como do bem-estar fetal, tais dificuldades podem ser desencadeadas pela liberação de cortisol e catecolaminas decorrentes do estresse doloroso (Mascarenhas et al, 2019). Assim, tais alterações hormonais podem modificar o processo de contração uterina e o fluxo placentário (Hillmann et al, 2019).

Tendo como base as possíveis intercorrências associadas ao processo doloroso do trabalho de parto, a analgesia tem sido uma prática cada vez mais discutida. Assim, as medidas não farmacológicas para o controle da dor são sempre as de primeira escolha, visto que as medidas farmacológicas podem apresentar efeitos adversos para o bebê, além de sedação excessiva para a mãe, dificultando a colaboração materna no processo do trabalho de parto (Dąbrowska et al, 2018). Os métodos farmacológicos para controle da dor do trabalho de parto podem ser administrados por via sistêmica (intravenosa, intramuscular ou inalatória) ou regional (bloqueio do nervo pudendo, analgesia espinhal e analgesia epidural). As técnicas neuroaxiais são o padrão ouro para o tratamento da analgésico intraparto atualmente, tendo como base sua maior segurança materno-fetal, além do alto potencial analgésico (Lausin et al, 2019). Epidemiologicamente, houve um aumento de cerca de 
$260 \%$ em 20 anos nos EUA, quando comparado os dados dos anos 80 e 90. No Reino Unido, uma a cada três gestantes foram submetidas ao bloqueio neuroaxial no ano de 2008 (Penuela et al, 2019).

Este artigo tem por objetivo avaliar a relação entre o uso das técnicas anestésicas e o desfecho obstétricos das gestantes durante o trabalho de parto, tendo como base os artigos publicados em base de dados.

\section{Metodologia}

Trata-se de uma revisão, cuja abordagem adotada foi quantitativa, que visa analisar e sintetizar o que a literatura traz sobre a analgesia do parto e seus possíveis desfechos obstétricos. Essa pesquisa levou em consideração os fundamentos de uma revisão integrativa de literatura, os quais consistem em: (1) identificação do tema e seleção da questão norteadora; (2) definição de critérios de inclusão e exclusão de estudos; (3) investigação na literatura; (4) estabelecimento das informações a serem coletadas dos trabalhos selecionados; (5) análise dos estudos selecionados; (6) interpretação dos resultados e (7) síntese das informações.

A busca de artigos foi feita nas seguintes bases de dados: Scientific Eletronic Library Online (SciELO) e no Literatura Latino-Americana e do Caribe (LILACS); além do portal PUBMED, utilizando os descritores: "Analgesia obstétrica", "Dor do parto" e "Trabalho de parto".

Na base SciELO, ao utilizar os três descritores em associação foram encontrados vinte e um artigos; ao usar o par "analgesia obstétrica" e "dor do parto" foram encontrados vinte e quatro artigos; utilizando o par "analgesia obstétrica" e "trabalho de parto" foram encontrados onze artigos; com o par "trabalho de parto" e "dor do parto" encontrou-se cento e treze artigos.

Na base LILACS, ao utilizar os três descritores foram encontrados quatro artigos; ao usar o par "Analgesia obstétrica" e "Dor do parto" foram encontrados dezoito artigos; utilizando o par "Analgesia obstétrica" e "Trabalho de parto" foram encontrados quarenta e oito. Na pesquisa dos descritores "Dor do parto" e "Trabalho de parto" foram encontrados trinta e quatro artigos.

No portal PUBMED, ao utilizar os três descritores foram encontrados duzentos e sete artigos; ao usar o par "Analgesia obstétrica" e "Dor do parto" foram encontrados duzentos e sete artigos; utilizando o par "Analgesia obstétrica" e "Trabalho de parto" foram encontrados quatrocentos e trinta e seis. Na pesquisa dos descritores "Dor do parto" e "Trabalho de parto" foram encontrados seiscentos e cinquenta e três artigos.

No total, foram encontrados mil setecentos e setenta e seis artigos na busca inicial, dos quais foram descartados mil setecentos e onze artigos por não estarem diretamente relacionados ao estudo. A seguir, sobraram sessenta e cinco artigos para leitura, excluindo-se quarenta e cinco por não adequação ao foco da discussão. Por conseguinte, foram selecionados vinte artigos. Destes foram excluídos sete por repetição. Os treze restantes foram realizados a leitura integral para a composição final desse estudo.

Os critérios de inclusão foram: artigos científicos disponibilizados de forma integral, que estivessem enquadrados como pesquisas em seres humanos com ano de publicação entre 2010 e 2020. Os critérios de exclusão foram: materiais que não fossem artigos científicos e estudos que não estivessem de acordo com o tema selecionado. 
Figura 1 - Fluxograma detalhando cada etapa do processo de seleção de artigos para a revisão.

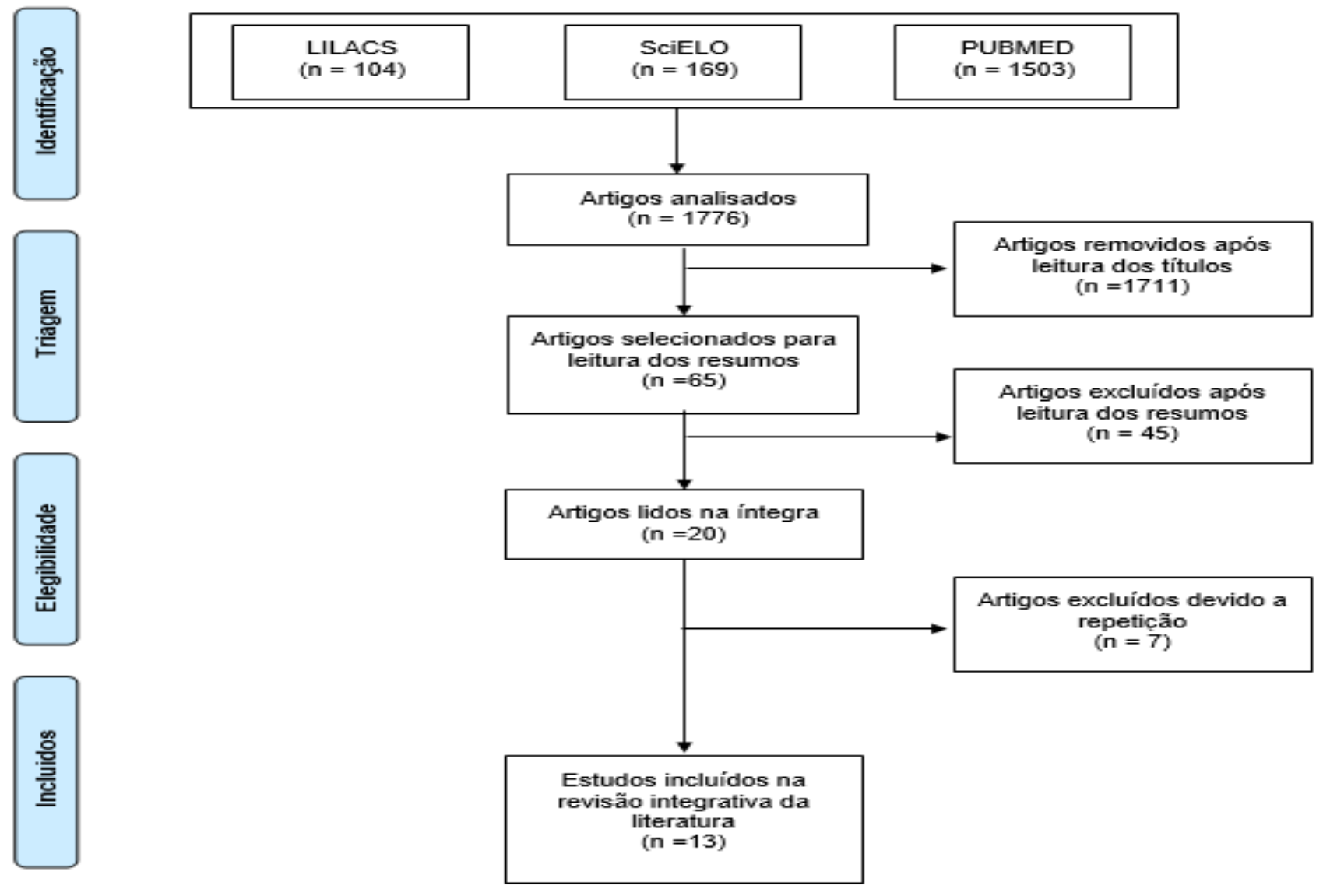

Fonte: Veiga et al. (2021)

O fluxograma da Figura 1 detalha cada etapa da revisão de literatura realizada: a coleta nas bases de dados consultadas (identificação), a seleção de artigos pela leitura do título e a leitura de seus resumos (triagem), a leitura de vinte artigos na integra após a triagem (Elegibilidade) e deste total, treze artigos utilizados para a composição do estudo que contemplavam os critérios de inclusão e que se adequassem a temática.

Quadro 1 - Lista de artigos selecionados para a composição do estudo, abordando a revista e ano de publicação e seus respectivos objetivos.

\begin{tabular}{|l|l|l|l|}
\hline Nome do Artigo & Revista & $\begin{array}{l}\text { Ano de } \\
\text { Publicação }\end{array}$ & Objetivos do Artigo \\
\hline $\begin{array}{l}\text { Labour analgesia and obstetric } \\
\text { outcomes }\end{array}$ & Br J Anaesth & 2010 & $\begin{array}{l}\text { O objetivo deste artigo é para revisar e resumir as evidências } \\
\text { disponíveis sobre o impacto da analgesia neuroaxial nos } \\
\text { resultados do parto e fornecer aos médicos uma compreensão } \\
\text { mais clara dos problemas }\end{array}$ \\
\hline $\begin{array}{l}\text { Relationship between Use of Labor } \\
\text { Pain Medications and Delayed Onset of } \\
\text { Lactation }\end{array}$ & J Hum Lact & 2014 & $\begin{array}{l}\text { Para investigar se o uso de medicamentos para a dor do parto } \\
\text { está associado a atrasos início da lactação (DOL). }\end{array}$ \\
\hline $\begin{array}{l}\text { Differential effects of epidural } \\
\text { analgesia on modes of delivery and } \\
\text { perinatal outcomes } \\
\text { nulliparous and multiparous women: a } \\
\text { retrospective cohort study }\end{array}$ & PLoS One & 2015 & $\begin{array}{l}\text { Os objetivos deste estudo foram estudar as características } \\
\text { demográficas e da gravidez maternas associadas ao uso } \\
\text { intraparto de analgesia peridural e investigar os efeitos da } \\
\text { analgesia peridural nos modos de parto e resultados perinatais } \\
\text { em uma grande coorte de ambas mulheres nulíparas e }\end{array}$ \\
\hline
\end{tabular}




\begin{tabular}{|c|c|c|c|}
\hline & & & multíparas. \\
\hline $\begin{array}{l}\text { Repercussões maternas e fetais da } \\
\text { analgesia obstétrica: uma revisão } \\
\text { integrativa }\end{array}$ & Av Enferm & 2015 & $\begin{array}{l}\text { Identificar as repercussões maternas e fetais do uso de } \\
\text { analgesias no trabalho de parto, relacionar as drogas mais } \\
\text { utilizadas, dosagens seguras e os critérios de indicação, bem } \\
\text { como analisar os efeitos da analgesia sobre a mãe e o feto. }\end{array}$ \\
\hline $\begin{array}{l}\text { Resultados obstétricos y perinatales en } \\
\text { pacientes con o sin analgesia obstétrica } \\
\text { durante el trabajo de parto }\end{array}$ & IATREIA & 2016 & $\begin{array}{l}\text { Descrever e comparar os resultados obstétricos e perinatais em } \\
\text { pacientes que recebeu analgesia obstétrica durante o trabalho } \\
\text { de parto com aquelas que não a receberam e determinar se a } \\
\text { referida analgesia está associada a resultados maternos ou } \\
\text { perinatais adversos. }\end{array}$ \\
\hline $\begin{array}{l}\text { Analgesia obstétrica farmacológica: um } \\
\text { estudo sobre os desfechos } \\
\text { obstétricos e neonatais }\end{array}$ & Rev Rene & 2017 & $\begin{array}{l}\text { Investigar a associação entre a analgesia obstétrica } \\
\text { farmacológica e os desfechos obstétricos e neonatais. }\end{array}$ \\
\hline $\begin{array}{l}\text { O uso de analgesia farmacológica } \\
\text { influencia no desfecho de parto? }\end{array}$ & $\begin{array}{l}\text { Acta Paul } \\
\text { Enferm. }\end{array}$ & 2017 & $\begin{array}{l}\text { Investigar associação entre analgesia farmacológica e } \\
\text { desfechos do parto. }\end{array}$ \\
\hline $\begin{array}{l}\text { A review of randomized trials } \\
\text { comparisons of epidural with } \\
\text { parenteral forms of pain relief during } \\
\text { labour and its impact on operative and } \\
\text { cesarean delivery rate }\end{array}$ & Ginekol Pol & 2018 & $\begin{array}{l}\text { O objetivo desta revisão foi resumir a literatura disponível } \\
\text { sobre as diferentes modalidades de alívio da dor do parto } \\
\text { (peridural vs. parenteral) e para avaliar seu impacto na taxa de } \\
\text { partos cesáreos e partos instrumentais, e sobre a necessidade de } \\
\text { apoiar a função contrátil uterina. }\end{array}$ \\
\hline $\begin{array}{l}\text { Evidências científicas sobre métodos } \\
\text { não farmacológicos para alívio da dor } \\
\text { do parto }\end{array}$ & $\begin{array}{l}\text { Acta Paul } \\
\text { Enferm }\end{array}$ & 2019 & $\begin{array}{l}\text { Identificar na literatura nacional e internacional, estudos sobre } \\
\text { a eficácia de métodos não farmacológicos na redução da dor do } \\
\text { parto. }\end{array}$ \\
\hline $\begin{array}{l}\text { Knowledge, attitude and practice } \\
\text { regarding pharmacological methods of } \\
\text { labor analgesia }\end{array}$ & BrJP & 2019 & $\begin{array}{l}\text { Avaliar o conhecimento, atitude e prática de obstetras sobre } \\
\text { métodos farmacológicos de analgesia de parto }\end{array}$ \\
\hline $\begin{array}{l}\text { Bloqueio combinado raquiperidural } \\
\text { para analgesia de parto. Estudo } \\
\text { comparativo com bloqueio peridural } \\
\text { contínuo }\end{array}$ & $\begin{array}{l}\text { Ver Bras } \\
\text { Anestesiol }\end{array}$ & 2019 & $\begin{array}{l}\text { Avaliar comparativamente a eficácia e a seguranc, a de duas } \\
\text { técnicas anestésicas: bloqueio combinado raquiperidural e } \\
\text { peridural contínua em grávidas submetidas à analgesia de } \\
\text { parto. }\end{array}$ \\
\hline $\begin{array}{l}\text { Relation between Length of Exposure } \\
\text { to Epidural Analgesia during Labour } \\
\text { and Birth Mode }\end{array}$ & $\begin{array}{l}\text { Int } \mathrm{J} \text { Environ } \\
\text { Res Public } \\
\text { Health }\end{array}$ & 2019 & $\begin{array}{l}\text { Tem como objetivo avaliar a relação entre o tempo de } \\
\text { exposição à analgesia peridural e o risco de nascimento não } \\
\text { espontâneo e para identificar fatores de risco adicionais. }\end{array}$ \\
\hline $\begin{array}{l}\text { Epidural analgesia and its implications } \\
\text { in the maternal health in a low parity } \\
\text { comunity }\end{array}$ & $\begin{array}{l}\text { BMC } \\
\text { Pregnancy } \\
\text { Childbirth }\end{array}$ & 2019 & $\begin{array}{l}\text { O objetivo deste estudo foi determinar a associação entre } \\
\text { analgesia epidural e diferentes complicações que afetam a } \\
\text { saúde materna, como SPT, parto instrumental, cesariana (CS) e } \\
\text { posição anormal da cabeça do feto no parto. }\end{array}$ \\
\hline
\end{tabular}

Fonte: Veiga et al. (2021)

Após o processo de seleção dos artigos para compor o estudo, a amostra final foi reunida no Quadro 1. Os artigos incluídos são de revistas diferentes e estão especificadas de acordo com: o título, o ano e o objetivo principal. 


\section{Resultados e Discussão}

A disponibilidade de estratégias para redução das dores no momento do parto não é tão aderida quanto o esperado no Brasil, mesmo após a garantia do direito das mulheres de ter alternativas para o manejo álgico conferido pelo Ministério da Saúde, Rede Cegonha e Diretrizes do Parto Normal. Diante desse quadro, em 2006, menos da metade das brasileiras foram possibilitadas de optar pelo controle da dor através de condutas não farmacológicas ou farmacológicas, quando as primeiras medidas são ineficazes no seu alívio. (Felisbino-Mendes, Santos, Amorim, Costa, \& Martins, 2017).

Apesar dos métodos anestésicos tornarem o trabalho de parto mais confortável, é importante considerar os riscos relativos para a saúde materna. Felisbino-Mendes et al. (2017), elenca como repercussões obstétricas adversas, o aumento do tempo do período expulsivo do feto, assim como da necessidade do uso de ocitocina para aumentar as contrações uterinas ou do uso do fórceps para exercer tração de expulsão.

Somado a isso, Piedrahíta-Gutiérrez, España-Chamorro, Piedrahíta-Gutiérrez, López-Clavijo, E Henao-Flórez, (2016), descreve os riscos relacionados a desfecho maternos e fetais adversos e maior taxa de cesariana. Os dados estudados revelam a existência da relação de fetos com parada cardíaca ou diminuição importante da frequência cardíaca fetal, critérios esses indicam as cesáreas de emergência, e a administração de fármacos anestésicos. Entretanto os estudos são insuficientes para afirmar absolutamente a presença dessa relação, considerando como viés o aumento da distócia de ombros como provável causa do aumento de indicações de parto cesáreo.

Os efeitos adversos maternos são considerados dose-dependentes, ou seja, quanto maior a dose administrada de anestésicos, maiores são os riscos de a genitora evoluir com hipotensão arterial, repercussões no córtex adrenal pelo aumento de noradrenalina e adrenalina, além das manifestações respiratórias, como a hiperventilação. No entanto, com os avanços dos estudos acerca da analgesia obstétrica, nota-se que a tolerabilidade do bloqueio é crescente o que reduz as possíveis complicações mais graves, conservando-se o prurido, a náusea e a, já citada, hipotensão. A diminuição da pressão arterial materna leva a uma diminuição do fluxo sanguíneo direcionado para o útero, gerando uma direta redução da motilidade uterina e sofrimento fetal por hipoxemia. Ademais, o prolongamento do período expulsivo do parto por mais de 3 horas cresce a probabilidade de parto cesáreo (Amaral et al., 2015).

Em relação a influência da analgesia no parto com o aumento da duração do trabalho de parto temos algumas controvérsias. Algumas literaturas alegam o aumento, diminuição ou até mesmo a não interferência da analgesia no tempo de trabalho de parto (Braga et al.2019). Porém, o que mais se destacou entre os estudos foi o aumento da duração do segundo período do trabalho de parto. Isso porque, apesar de satisfatória para controlar a dor, a administração de anestésicos locais pode desencadear diversos efeitos indesejáveis. Dentre eles, o relaxamento da musculatura do assoalho pélvico e da parede abdominal e uma hipotensão secundária ao bloqueio simpático na gestante, desencadeando uma tribulação na rotação interna do polo cefálico do feto no canal do parto juntamente com uma anulação do reflexo de Ferguson. Tais eventos proporcionam o aumento da duração do período expulsivo e uma consequente elevação do número de partos instrumentais. (Cecatti et al.1998)

Acerca do parto vaginal instrumental, o The American College of Obstetricians and Gynecologists (2015, como citado em Felisbino-Mendes et al., 2017, p.459), o define como aquele que utiliza o fórceps ou vácuo extrator para auxiliar no período expulsivo do parto. As indicações, como citado em Nikpoor e Bain (2013), são condições maternas e fetais que podem causar danos ao vetor mãe-filho e devem ser utilizados no segundo tempo do trabalho de parto. Porém, muitos estudos atuais estão apontando riscos dessa prática, dentre elas, morte materna e fetal.

Nos artigos analisados para a pesquisa, percebeu-se que há uma relação direta entre o aumento da realização de parto vaginais instrumentais e uso de analgesia farmacológica. Alguns apresentaram, ainda, fatores de risco, associado com analgesia, que aumentaram os índices desse tipo de parto. A maioria das pesquisas estudaram especificamente a relação com a 
Analgesia Epidural (AE), uma vez que esta é considerada um dos métodos de alivio da dor mais eficaz durante o trabalho de parto (Hung, Hsieh, \& Liu 2015).

No estudo Felisbino-Mendes et al, (2017) foi identificado que mulheres que receberam algum tipo de analgesia farmacológica, ou seja, utilizou de fármacos para supressão da dor, tiveram quase quatro vezes a chance de ter um parto vaginal assistido. No que tange somente a utilização de analgesia epidural, Anim-Somuah, M., Smyth, R. M., Cyna, A. M., \& Cuthbert, A. (2018, como citado Garcia-Lausin et al., 2019, p.2) concluiu que existe um risco absoluto de 14,2\% de parto vaginal instrumental quando se administra $\mathrm{AE}$.

Alguns autores estabeleceram uma associação entre fatores de risco para parto com utilização de fórceps ou vácuo extrator. Na pesquisa de Hout et al (1977, citado em Mędrzycka-Dąbrowska, Czyż-Szypenbejl, \& Pietrzak 2018), os resultados corroboram com os acimas citados e apontaram que a utilização de analgesia durante o parto causa o aumento do parto assistido, porém traz uma incidência de $40 \%$ em multíparas e $70 \%$ em nulíparas. Além disso, não há evidências que a administração precoce, ou tardia, altere esse índice. (Fernandes, Damasceno, Herculano, Martins, \& Oriá, 2017)

Referindo-se à amamentação do RN, é consenso na literatura a importância da amamentação exclusiva do lactente até 6 meses de idade e de início logo na primeira hora de vida. Isso se justifica pois, além dos benefícios a longo prazo, como prevenção da obesidade e da diabetes mellitus tipo 2, esse leite é provedor de nutrientes necessários para o bom desenvolvimento do RN, além de conter anticorpos que auxiliam na proteção contra agentes infecciosos causadores de doenças como diarreia e pneumonia, duas das principais causas de morte de crianças no mundo (Organização Mundial da Saúde, 2018).

No entanto, é importante atentar para os riscos do atraso da lactação causado pelo uso de medicamentos que aliviam a dor no trabalho de parto principalmente por meio do bloqueio epidural. $\mathrm{O}$ atraso do início da lactação (DOL na sigla em inglês) se caracteriza pelo início da lactação abundante mais 3 dias pós-parto, o uso da analgesia durante o parto pode aumentar entre 2 a 3 vezes as chances lactação tardia quando comparadas com pacientes que não receberam medicamentos para dor do parto e o fizeram de forma vaginal (Lind, Perrine \& Li, 2014).

De acordo com o artigo Nursing protocol for assistance to women in lactation process, de 2017, a administração de analgesia epidural durante o trabalho de parto, assim como o estresse materno, está relacionada com a diminuição dos níveis séricos de ocitocina, hormônio importante para a lactação e ejeção do leite contido nas glândulas mamárias. Como principal repercussão clínica, tem-se o desmame precoce, com média de 3,4 meses de amamentação exclusiva para filhos de lactantes com DOL contra média de 11.7 meses para RNs que iniciaram o leite materno com até 3 dias de atraso. (Lind et al., 2014).

No que tange a saúde fetal, algumas repercussões foram observadas nos artigos analisados. Apesar de sua etiologia incerta, a bradicardia fetal relaciona-se com a cessação abrupta da dor das contrações uterinas que resulta no desequilíbrio plasmático de epinefrina e norepinefrina, ocasionando diminuição da oferta sanguínea uteroplacentária. Esse efeito foi observado com a administração de clonidina via subaracnóide e bupivacaína sem vasoconstritor associado ao fentanil via peridural (Amaral et al., 2015). Os autores Hung et al., (2015) também apontaram maior frequência deste fenômeno em gestantes nulíparas submetidas a analgesia epidural e relacionaram a analgesia à prevenção de ansiedade e dor, fatores que contribuem com a ocorrência de bradicardia fetal.

Outro fator presente foi a acidose fetal, analisada pelos autores Amaral et al., (2015), a qual acredita-se que seja ocasionada devido ao prolongamento do segundo estágio do trabalho de parto. O uso de bupivacaína isolada em diferentes concentrações relaciona-se a este efeito adverso. No entanto, o uso de bupivacaína associado ao sufentanil viabiliza menores doses do fármaco para o mesmo efeito analgésico, permitindo a redução dos danos materno-fetais.

No âmbito da saúde neonatal, um dos parâmetros utilizados para avaliar o estado do recém-nascido e a resposta à ressuscitação é o escore APGAR. Os autores Piedrahíta-Gutiérrez et al., (2016) pontuaram que a analgesia obstétrica não se relaciona com APGAR menor que 5 no primeiro minuto, menor que 7 no quinto minuto, necessidade de reanimação neonatal 
ou internação em UTI. Em concordância, uma maior probabilidade de escore menor que 7 no primeiro minuto foi notada nos estudos de Fernandes et al., (2017) e Hung et al., (2015). Não houve diferença significativa no quinto minuto entre os escores dos bebês de mulheres com analgesia e sem analgesia (Fernandes et al., 2017).

\section{Considerações Finais}

O momento do parto é um momento único para a parturiente e para o bebê. Durante esse período o corpo da mulher receberá diversos estímulos para a expulsão do feto, inclusive o da dor - o qual é diferente para cada indivíduo. Por isso, deve ser sempre respeitado e garantido o direito da mulher ao acesso a analgesia. Não somente no momento do parto, mas também durante a gestação, deve-se informar a mulher sobre como ocorrerá o parto e as ferramentas que a medicina possui para o manejo da dor (farmacológicas e não farmacológicas). Este artigo considerou que há o benefício da utilização de analgesia no alivio da dor e conforto durante o parto, porém observou-se que com sua utilização há o aumento do número de parto vaginais instrumentais, aumento de 2 a 3 vezes nos casos de DOL, em alguns casos de AE ocorre bradicardia fetal e ainda aumento do segundo estágio do trabalho de parto (este ainda relacionado com acidose fetal). Acerca das cesarianas foi vista uma relação com a analgesia, porém os estudos analisados consideram insuficientes. Diante do exposto, é necessário alertar a gestante para os benefícios e os riscos da prática, assim a parturiente poderá fazer uma escolha consciente da utilização ou não de medicamentos para alívio da dor.

Além disso, fomenta-se o desenvolvimento de novos estudos acerca da analgesia e o aumento de parto cesáreo com resultados mais claros e consistentes, a fim de elucidar se há a relação entre eles. Desse modo, os profissionais de saúde, por meio de embasamento cientifico, podem orientar da melhor forma a gestante.

\section{Referências}

Amaral, H. R. M., Filho, E. D. S., Silva, D. M., Barbosa, T. L. d. A., \& Gomes, L. M. X. (2015, June 15). Repercussões maternas e fetais da analgesia obstétrica: uma revisão integrativa. Av Enferm, 33(2), 282-294.

American Academy of Pediatrics, Committee on Fetus and Newborn, American College of Obstetricians and Gynecologists, \& Committee on Obstetric Practice (2006). The Apgar score. Advances in neonatal care: official journal of the National Association of Neonatal Nurses, 6(4), $220-223$.

Braga, A. F. A., Carvalho, V. H., Braga, F. S. S., \& Pereira, R. I. C. (2019). Bloqueio combinado raquiperidural para analgesia de parto. Estudo comparativo com bloqueio peridural contínuo. Revista Brasileira de Anestesiologia, 69(1), 7-12. https://doi.org/10.1016/j.bjane.2018.08.003.

Cecatti, J. G., Pereira, R. I. C., Oliveira, A. S., \& Cecarelli, M J. (1998). Analgesia peridural para o trabalho de parto e para o parto: efeitos da adição de um opióide. Revista Brasileira de Ginecologia e Obstetrícia, 20(6), 325-331. https://doi.org/10.1590/S0100-72031998000600005.

Dąbrowska W. M., Szypenbejl K. C., Pietrzak J, et al. A review of randomized trials comparisons of epidural with parenteral forms of pain relief during labour and its impact on operative and cesarean delivery rate. Ginekologia Polska. 89(8), 459-466.

Felisbino-Mendes, M. S., Santos, L. O., Amorim, T., Costa, I. N., \& Martins, E. F. (2017). O uso de analgesia farmacológica influencia no desfecho de parto? Acta Paul Enferm, 30(5), 458-464.

Fernandes, R. L. V., de Castro Damasceno, A. K., Herculano, M. M. S., Martins, R. D. S. T., \& Oriá, M. O. B. (2017). Analgesia obstétrica farmacológica: um estudo sobre os desfechos obstétricos e neonatais. Revista da Rede de Enfermagem do Nordeste, 18(5), 687-694.

Hillmann B. R., Stamm A. M. N. F, et al. Knowledge, attitude and practice regarding pharmacological methods of labor analgesia. BrJP. 2(1):14-9.

Hoult I. J, MacLennan A. H, Carrie L. E. Lumbar epidural analgesia in labour: relation to fetal malposition and instrumental delivery. Br Med J. 1977; 1(6052): 14-16.

Hung, T. H., \& Liu, H. P. (2015). Differential effects of epidural analgesia on modes of delivery and perinatal outcomes between nulliparous and multiparous women: a retrospective cohort study. PLoS One, 10(3), e0120907.

Lausin L. G, Botella M. P, Duran X, Vicente M. F. M, Martin M. J. G, Cuesta E. G. E, Escuriet R., et al. Relation between Length of Exposure to Epidural Analgesia during Labour and Birth Mode. Int. J. Environ. Res. Public Health 2019, 2916-2928.

Penuela I., Nebreda P. I., Almeida H., López M., Sanchez E. G., Tamayo E., et al. Epidural analgesia and its implications in the maternal health in a low parity comunity. BMC Pregnancy and Childbirth. 2019;19:52. 
Research, Society and Development, v. 10, n. 3, e25410313307, 2021

(CC BY 4.0) | ISSN 2525-3409 | DOI: http://dx.doi.org/10.33448/rsd-v10i3.13307

Pereira, A. S., et al. (2018). Metodologia da pesquisa científica. UFSM. https://repositorio.ufsm.br/bitstream/handle /1/15824/Lic_Computacao_MetodologiaPesquisa-Cientifica.pdf?sequence=1

Piedrahíta-Gutiérrez, D. L., España-Chamorro, J. A., Piedrahíta-Gutiérrez, W. E., López-Clavijo, C. A., \& Henao-Flórez, R. E. (2016, September 1). Resultados obstétricos y perinatales en pacientes con o sin analgesia obstétrica durante el trabajo de parto. IATREIA, 29(3), 263-268.

The American College of Obstetricians and Gynecologists. Operative Vaginal Delivery. Obstet Gynecol. 2015; 126(5): e56-e65.

Vieira G. M., Morais T. B., Lima E. F. A., Pontes M. B., Brandão M. A. G., Primo C.C. Nursing protocol for assistance to women in lactation process. Rev Fun Care Online. 9(4): 1040-1047. DOI: http://dx.doi. org/10.9789/2175-5361.2017.v9i4.1040-1047

World Health Organization. 10 facts on breastfeeding. http://www.who.int/features/ factfiles/breastfeeding/facts/en/index9.html. Mascarenhas, V. H., Lima, T. R., Silva, F. M., Negreiros, F. S., Santos, J. D., Moura, M. A., et al. Evidências científi cas sobre métodos não farmacológicos para alívio a dor do parto. Acta Paul Enferm. 2019;32(3):350-7. 